\title{
Criteria for Rapidly Incorporating Online Instruction into Traditionally In-Person Programs: A case study
}

\author{
Steven C. Grambow, PhD \\ Department of Biostatistics and Bioinformatics, \\ Duke University Medical Center, Durham NC, United States \\ Gregory P. Samsa, PhD \\ Department of Biostatistics and Bioinformatics, \\ Duke University Medical Center, Durham NC, United States
}

\begin{abstract}
Given the continuing uncertainty about the COCID-19 pandemic, we are planning for the possibility that, in the upcoming semester, some or all of the content of a Master of Biostatistics program will be delivered online. In order to help make this rollout as systematic as possible, we propose criteria which an effective online-enabled educational program should meet. We will incorporate online instruction into our program when: (1) it is necessary and/or educationally beneficial; (2) it can be delivered with sufficient technical quality; (3) instructors have been trained to deliver it sufficiently well; (4) both content and delivery undergo regular review; and (5) policies are in place to support online instruction. These criteria are not unique to graduate programs in biostatistics and, thus, we are presenting this short report and case study in the hope that it will be beneficial to others as they consider and/or prepare for transition to online instruction.
\end{abstract}

Keywords: Curriculum development; Online instruction; Program assessment.

\section{INTRODUCTION}

We are responsible, in our roles as Associate Chair for Education and Director of Graduate Studies, for (among others) overseeing a Master of Biostatistics program. Classes are delivered in person, and the program utilizes a learning management system [1] which performs tasks such as placing syllabi, class notes, and datasets online. The program can thus be classified as "in-person delivery, with some support functions performed online".

Like many graduate programs, we were required to urgently respond to the COVID-19 pandemic, and the core of that response was to rapidly move all our classes to synchronous online delivery. The move took place within a two-week period (one week of spring break plus another week for additional preparation). Essentially, our classes were structured as before - or, at least, as close an approximation as we could achieve given the quick turnaround - but were delivered to a physically disparate audience using the Zoom videoconferencing platform [2], available to all students and faculty at our institution. 
Given the continuing uncertainty about the pandemic, we are planning for the possibility that, in the upcoming semester, some or all of our classes will be delivered online to some or all of our students. With more time to prepare and some experience under our belt, our intention is to do so as systematically as possible and, indeed, to embed within the development process reassurance that our implementation of online instruction is educationally sound. In designing a development process, a first step was establishing various criteria which an effective online-enabled educational program should meet.

These criteria are not unique to graduate programs in biostatistics and, thus, we are presenting this short report and case study in the hope that it will be beneficial to others as they consider and/or prepare for transition to online instruction.

\section{CRITERIA}

We will incorporate online instruction into the Master of Biostatistics program when:

- It is necessary and/or educationally beneficial

- It can be delivered with sufficient technical quality

- Instructors have been trained to deliver it sufficiently well

- Both content and delivery undergo regular review

- Programmatic policies are in place to support online instruction

\section{Necessity}

For planning purposes, we anticipate a significant likelihood that some or all of our students will be unable to attend fall classes in person. If our institution decides to temporarily eliminate in-person classes during the fall, this will apply to all students. If international students currently residing outside this country are prevented from entering the country due to travel restrictions, encounter delays in processing/receiving visas, or are required to self-quarantine entering the country, this will apply to some students, at least for some period of time.

\section{Educational benefit}

The rationale for online instruction is not simply to approximate an in-class experience, to a greater or lesser degree of fidelity. Some educational tasks may, in fact, be better performed online (or using a combination of in-person and online instruction). For example, we already use a learning management system to perform tasks such as placing syllabi, class notes, and datasets online, and this can be usefully expanded to include videos of class sessions or step-by-step homework solution walkthroughs. Such videos can assist students in reviewing materials and, indeed, an advantage in comparison with an in-person class is that students can control the flow of the videos, replay sections of the video as needed, etc.

Another task which may be better performed online is conversation about course material. Chat messaging is a key tool in synchronous online courses, enabling conversations to take place before, during and after class, without disrupting the flow of the class. As a practical recommendation, if chat is to be significantly integrated into instruction, it is beneficial to have someone other than the instructor, a co-instructor or teaching assistant perhaps, monitor the chat for questions and comments from students. These "backchannel" conversations can help increase participation in 
Grambow, S. C., \& Samsa, G. P. (2020) Criteria for Rapidly Incorporating Online Instruction into Traditionally In-Person Programs: A case study. Advances in Social Sciences Research Iournal, 7(4) 359-364.

classroom discussions and provide an informal assessment of student comprehension of the course materials being presented.

Within biostatistics, one area of instruction that may be particularly well suited to synchronous online teaching is statistical computing and data analysis. More specifically, live software and coding demonstrations provide students with the opportunity to view the instructor's screen from a first-person perspective. Screen sharing functions present in many video conferencing platforms allow students encountering difficulties to share their screen with the instructors to receive help in real time. In this and similar contexts, synchronous online instruction may help increase engagement, reduce student frustration, and increase student comprehension.

\section{Technical quality}

Although technical quality is critical in any event, it is most challenging for synchronous online instruction (e.g., "holding class online") rather than asynchronous online instruction (e.g., archiving materials online for self-study). Potential problems include a lack of the desired functionality (e.g., the software used to support online instruction might not do everything that is desired), interruptions to the online experience (e.g., the software crashes, poor audio quality, digital lag and delay), and the possibility of malicious hacking (commonly known as "zoom bombing").

A key component to technical quality is adequate technical staffing. For our program, technical experts (among others) provide advice about the choice of video conferencing equipment (e.g., microphones, webcams) and other online learning tools (e.g., polling software, drawing tablets, etc.). They hold training sessions for both students and faculty. They also facilitate online sessions, including scheduling, meeting configuration, hosting, and real time troubleshooting.

\section{Instructor training}

Although in an emergency an instructor can muddle through by simply pretending that their students are in a physical classroom rather than disembodied faces on a computer screen, this is not the optimal way to proceed. Training is needed. While some technical training is needed to interface with the video conferencing platform and run the class sessions, the ideal trainer isn't necessarily someone who is trying to extend the cutting edge, either as a highly innovative educator or as a digital learning consultant - someone whose message is "you might this try this bell and you might try this whistle, they haven't been fully tested but seem quite interesting" - but rather someone with both expertise in teaching biostatistics and knowledge about online teaching someone whose message is "I found it helpful in my teaching, it works, and once you've taken the time to learn it using it won't be a burden".

Many of our upcoming fall course instructors did not teach during this past term, have been somewhat insulated from the details of this rapid transition, and have little/no experience teaching online classes. However, our program now has a new cadre of experienced teachers who can provide practical advice, and has also accumulated experience in training instructors (from scratch, if necessary).

\section{Necessity}

A critical component of any educational program is review, and online instruction is no exception. Indeed, both the magnitude of the change and the high stakes suggest that review should be 
extensive and ongoing. Instructors should be consulted as review criteria are selected, and a possible question to start the discussion might be "What should we monitor to know whether online instruction is going well (or poorly), and thus what modifications are urgently needed?". Including student input into these deliberations has much to recommend it.

Online instruction also opens up the possibility of additional peer review, as other faculty members can essentially "sit in the back of the class", either in real time or after the fact, without the potential awkwardness and distraction of physically doing so.

\section{Necessity}

Programmatic changes of this magnitude should be supported by the infrastructure of written program-level policy. These policies would provide answers to questions such as:

- Which courses are best suited to being delivered online?

- Is there a limit on the number of online courses a student can take?

- Which examinations should (and should not) be moved online?

- How should online examinations be proctored?

- How will online instruction be evaluated?

- How do program structure and policies maintain compliance with university, accreditation, and international student regulations?

Policies can be considered tentative, with the expectation that they might be revised as additional experience accumulates, but should be developed as early as possible as they provide a formal structure to increase the likelihood of success. Such a structure can also provide university administration and accreditation agencies with a record of what is intended (and delivered), thus generating a continuous information stream that ensures compliance with relevant standards.

\section{DISCUSSION}

The regular operations of schools and universities worldwide have been disrupted by the current pandemic. This has forced a rapid transition to technology-based online approaches to teaching and learning. While the long-term impacts of this unplanned global experiment on educational pedagogy are uncertain, there remains a practical need to be prepared for continued disruption in the coming months.

We have discussed some criteria which an effective online-enabled educational program should meet, and also illustrated how these criteria are interfacing with the process for systematically rolling out additional elements of online instruction within a graduate program.

Our primary focus in this report has not been the details of the "acute-phase reaction" to the pandemic, but instead upon criteria that might be applied for systematically managing the transition to online instruction going forward. Nevertheless, a few reflections about the transition to date might be in order. Perhaps most importantly, our paramount concern through this process has been the academic and emotional well-being of our students. For example, our students typically tend to congregate in groups to study and share information about class content. Because our state government implemented a stay-at-home order during this period, we were concerned 
Grambow, S. C., \& Samsa, G. P. (2020) Criteria for Rapidly Incorporating Online Instruction into Traditionally In-Person Programs: A case study. Advances in Social Sciences Research Iournal, 7(4) 359-364.

about the impact of the resulting social isolation. As a result, we facilitated virtual student meetings and provided information about university resources around emotional support.

Our approach to addressing the impact of the transition on academic outcomes has included tutorials about how to join online class sessions and the basic communication tools used during those sessions. Our university also provided extensive online tutorials, live virtual office hours, and live virtual training sessions for both students and faculty. In addition, we have modified our traditional end-of-term course evaluations to gather information about the impact of the transition on student learning as well as seeking feedback on specific teaching strategies and technology elements utilized in online classes. We continue to explore how grading can be best accomplished in the online environment.

Beyond the current crisis, graduate education is undergoing sweeping changes as, for example, students (and their parents) are increasingly questioning the value they receive in comparison to its costs. Online instruction is a part of those changes, and the challenges include determining "when" and "how". One barrier has simply been energy of activation, and that barrier has been obliterated by the pandemic. Necessity truly is the mother of invention.

In that spirit, some sources of optimism are evident. As discussed above, we anticipate that incorporating some elements of online instruction into our curriculum will be educationally beneficial in any event, and might be retained when (and if) our program returns to being primarily delivered in person. Moreover, although the amount of initial preparation time is extensive, the net result is often that classes are better prepared, and expectations about the organizational level and quality of instruction are correspondingly raised. A video record of classes is also generated, thus facilitating review of how well those heightened expectations have been met. Finally, the various discussions among faculty members, instructional designers, support staff and others has served to increase discussion and raise awareness about effective teaching practices, not to mention being an interesting and collegial experience.

Despite the current chaos, we remain optimistic about the potential of online instruction, and hope that these reflections on our experiences will be helpful to others.

\section{References}

[1]. https://www.sakaims.org/

[2]. https://zoom.us/ 